\title{
Endocrine and Behavioural Response of Dog in Stress Conditions
}

\author{
Natalia ȚURCANU ${ }^{1}$, Ionel PAPUC ${ }^{1}$ \\ 1) University of Agricultural Sciences and Veterinary Medicine, Faculty of Veterinary Medicine \\ Department of Semiology, Radiology and Medical Imaging, Mănăștur, no. 3-5, Cluj Napoca, Romania; \\ *corresponding author: papuc_i@yahoo.com
}

Bulletin UASVM Veterinary Medicine 73(2) / 2016,

Print ISSN 1843-5270; Electronic ISSN 1843-5378

DOI:10.15835/buasvmcn-vm: 11967

\begin{abstract}
Visit the veterinarian for different reasons constitutes an unpleasant/negative or perhaps stressed experience for dog. The stress could modify the physiological function and mask the symptom of an illness, or make the dog aggressive.

Comparing the endocrine and behavioral response of dogs during the visit to the veterinary hospital and during activities in other places that are not related to stress (workplace, park). Six dogs were taken in consideration, as indicators of stress there were used urinary cortisol, and behavioral observations.

Four of six dogs got high values of urinary cortisol after the visit to the veterinary clinic comparing with the values obtained in working spaces/park - areas that were familiar for dogs. Behavioral data's have indicated a hesitating behavior at the entrance of the clinic and also in the examination room for all dogs, but the levels of intensity range individually for each dog. The results of the „Clinic Dog Stress Scale” classify behavior of dogs in following categories: 1 dog had score of 1 point that means a calm, relaxed dog; 2 dogs- 3 points that means a middle level of stress, the dog is tense but cooperative; 2 dogs got 4 points-very tense, difficult to maneuver, and one dog 5 points that means a high level of distress.

Studding behavior relating with stress and the endocrine response during the visit to the vet clinic has importance for animal, owner and veterinarian. There is also a correlation between the stress manifestation and urinary cortisol.
\end{abstract}

Keywords: dogs, observation of behavior, urinary cortisol

\section{INTRODUCTION}

Scientific research attests that the welfare of the animal is influenced by several factors, such as: genetic predisposition, gender, age, life experiences with which the animal was confronted. Facing new situations, unpredictable for the animal, constitutes stressor that can cause certain behaviors that threaten both animal life as well as those who are around him.

A major role in ensuring an optimal level of human-dog interaction is played by veterinarian. Choosing a veterinarian is based on various aspects such as: specialization, distance to the clinic, schedule, sympathy for the doctor, vet's skills, cost of consultation or treatment, quality of offered services, vet behavior regarding the companion animal, the positive attitude towards it, etc. (Rusu, 2012).

Visit to the vet could be associated by the dog with a frustrating and often painful experience. This is easy to guess from dog behavior but also by changes in the hormonal status of the body. The importance of this problem is multifaceted and refers to animal welfare, the safety of the handler and clinical stuff, additional costs and owner opinion about the clinic.

Studies show that $60 \%$ of dogs brought to the clinic for a routine examination showed 
an anxious behavioral and obedience. In 1981, Stanford mentions that around $70 \%$ of the dogs that were brought to the vet showed a hesitant or refusal behavior to entry into the clinic, from those, $60 \%$ presented clearly anxious behavior, $18 \%$ expressed their fear biting and 5\% had other defensive behaviors (those can be forms of aggression but so does the tendency to escape). A more recent study obtained similar results, with $78.8 \%$ of dogs show behaviors that betray fear, especially during examination (Doring et al, 2009).

Visit to the vet for different reasons constitutes an unpleasant/negative or perhaps stressing experience for the dog. The stress could modify the physiological function and mask the symptom of an illness, or make the dog aggressive (Jensen, 2007).

The aim of this study was to compare the endocrine and behavioral response of dogs during the visit to the veterinary hospital with the dog's activities in other places that are not related to stress (workplace, park).

\section{MATERIAL AND METHOD}

In the present study 6 dogs were selected. Dog's participation in this project was done in collaboration with the owners, after their take knowledge about the reason and conditions of the study. The area from where the owners were selected comprising also the area where they take their animals usually. The dogs were selected, taking into consideration the following criteria: age, sex, state of health of the animal and whether or not they suffer an ovariohysterectomy operation. Based on these criteria were selected: 6 adult females between 3 and 10 years old, who underwent ovariohysterectomy intervention. The health of the animal was assessed after anamnesis in which the owner declared that that the animals did not suffer conditions that influence cortisol values.

The experimental design of the survey was divided into three phases:

1. Collecting and sending urine samples to the laboratory;

2. Observing the behavior of the dogs in the three stages of the visit to veterinary clinic (when traveling to clinical, time spent in vet clinic and departure from the vet clinic);
3. Quantification of stress level rating scale based on the level of stress in the veterinary clinic

Urine from each dog was collected during spontaneous micturition. According to the following protocol: 2 urine samples were collected during 7.00-10.00 am. The first sampling or collect, took place in walking area of the dog place known by animal and the second was done after the visit to the veterinary clinic respecting same time interval.

Visit to the clinic lasted about 15 to 30 minutes urine collection was carried out after 30 minutes 1 hour after leaving the clinic.

Disposable clean containers (urocultor) where used, provided with tight lid so as to prevent spills, evaporation or contamination, $2 \mathrm{ml}$ of urine was collected. The urine container was identified by name, breed, age of the animal and where the harvest of urine was performed. Storage and transport were performed following the classical protocols.

\section{Determination of cortisol}

Laboratory measurements were made in the Synevovet laboratory and reference values for cortisol were provided by the same laboratory. Laboratory method used to determine the concentration of urinary cortisol - with detection by chemiluminiscence immunoassay. Urinary cortisol was chosen to be determinate because it present the followings advantages: it is a noninvasive method, it is simple and it requires no extra stressful sampling procedure that would influence the cortisol level compared with measurements of plasma cortisol (venipuncture) or saliva cortisol (food contamination).

\section{Behavioral observations}

Visit to the veterinary clinic consisted of completing the route to the clinic, for those who have been there being a known route, and the dog can possibly link the way to the clinic with a negative / positive experience. For those who do not associate the route with the veterinary clinical this represents another new unknown trail. Owners were asked to enter with the animals into the waiting room and then into the examination room without intervening in any particular form. The dog was accompanied by the owner throughout their stay in the clinic. Although the visit has not been with a diagnostic or therapeutic purpose, the presence of unknown people, other 
animals, and a new and unsecured environment mimicked frequent situation for pet.

Observing the behavior of dogs took place, both during walks in special areas for walking the dogs and at the veterinary clinic.

During walking in specially designated areas or areas where dogs were accustomed the interaction master - dog was observed and the type of rewards (verbal, favorite toy or food).

Tracking animal behavioral reactions during the visit begin during the movement toward clinic, throughout their stay in the clinic and walk from the clinic. Upon observation were targeted the following: behaviors displayed when traveling to clinic: behavior suggesting reconnaissance towards the clinic, refusing to go to the clinic or absence of clinical behavioral cues that would indicate refusal, owner's reaction to these behaviors (positive or negative reinforcement). Behavioral manifestations in the clinic interaction with other animals, people (presence of other owners, clinic staff). Dog's behavior out of the clinic (behavior that betrays the desire of departure, restlessness, anxiety). It has taken into account the descriptions provided by the owner of the dog's previous experiences related to doctor visits. A scale was used for assessing the stress level (Overall, 2013).

Rating Scale was used to assess the level of stress during the stay in the waiting room and examination room. Dogs with a score of 5 points are considered as having high levels of stress and need help. Dogs with 0 points score are valued as calm / quiet. Assessing the level of stress when traveling to clinic was made only by observing the behavior / attitudes / body expressions reported to a relaxed behavior (Table 1).

\section{RESULTS AND DISCUTION}

The results obtained reflectcortisol differences in the two contexts: dogs in the walking area (area known to dog) and after visiting the veterinary clinic (Table 2).

The results show clearly that the visit to the veterinarian is a situation that calls for the animal to adapt to new stimuli through both behavioral and neuroendocrine mechanism.

Individual variations of urinary cortisol and behavior is explained by the previous experiences of the dog during the visit to the veterinarian environment (past medical interventions, the time since the last visit), the dog's personality but also the verbal and nonverbal messages the owner send or factors that related to the veterinary clinic (waiting room and treatment area).

The difference in urinary cortisol from urine samples obtained in the park or dogs walking space, those spaces being associated with play, rewards and socializing with other dogs, and urinary cortisol values from urine samples obtained in the veterinary clinic indicates that although unequally entry into a new room, the smell is perceived as threatening the safety of the dog, translating into physiological terms by activating warning systems (CNS, PNS, endocrine system) or increase the urinary cortisol levels.

Observations on the behavior of dogs, regarding the three sequences linked to the veterinary clinic, correlated with urinary cortisol values obtained after leaving the clinic suggests that animals that have a high score on the scale of increased stress assessments have also high urinary cortisol level. Namely dogs with low stress score values were show lower cortisol level after visiting the veterinary clinic.

Tab. 1. Assessment scale for stress in the veterinary clinic (Overall, 2013)

\begin{tabular}{cc}
\hline Stress level & Dog behavior / expression / attitudes; \\
\hline 0 points & Very friendly, interact, ask for attention; \\
\hline 1 point & Calm, relaxed, seemingly affected / uninterested; \\
\hline 2 points & Alert but calm and co-operative; \\
\hline 3 points & Tense but cooperative, not very relaxed but can still be easily controlled on a leash; \\
\hline 4 points & $\begin{array}{r}\text { Very tense - manifest obvious anxiety, trembling, vocal sounds (yelp), } \\
\text { do not obey commands, difficult to handle with a leash; }\end{array}$ \\
\hline 5 points & $\begin{array}{c}\text { Very nervous, barking / howling, trying to hide, it needs to be raised } \\
\text { or forced when pull the leash or refuse to enter the clinic. }\end{array}$ \\
\hline
\end{tabular}


The non-homogeneity of the group assessed in terms of age and sex (adult females) does not allow comparison with other groups based on these criteria but reported to other studies (Doring, 2009; Kolevska et al., 2003; Kelsey, 2012), based on our observation we can say that adult dogs present a high level of anxiety compared to those younger than 2 years. Regarding sex of animals, studies are conflicting, no correlations were found between animals sterilized and anxiety levels at the veterinary clinic, but it has been established that the males provide a level of fear or fear much lower than females (Moberg and Mench, 2000; Marcus, 2007; Mills, 2013).

Increased levels of cortisol in urine samples after a visit to the veterinary clinic can be correlated with the combination of negative experience (ovariohysterectomy) and office / veterinary staff, plus the number of other painful treatments.

The results indicate behavioral differences (moving towards the clinic) between animals that have been to the clinic before the study (2 dogs-that where operated in the clinic where the study was made), compared to those who were not (4 dogs who visit the clinic where the study was made, for the first time). Dogs that went to the clinic before recognized the way and as they approach to the clinic became more restless and refused to move. Five of six dog's behavior was hesitant at building entrances. Stanford (1981) noted that many dogs do not enter voluntarily in veterinary clinic exhibiting behavior that indicates fear and $70 \%$ of the animals were forced into the waiting room. Doring (2009) notes that $13 \%$ of dogs have the same behavior when entering the examination room.

The lower urinary cortisol after clinic visit compared with the values of cortisol in the park, correlated with the nonverbal language e of the owner, which conveys fear, distrust of the clinical experience, is reflected in the dog behavior. Adopting a neutral attitude by owners is reflected in dog's behavior when travelling to clinic, in the waiting room, in the treatment room and also in the urinary cortisol values.

A calm and relaxed behavior in all stages of the study and low urinary cortisol can be explained by the relationship of trust between owner and dog (frequent verbal encouragement, rewards food, ball), dog's personality; time spent together (hiking).

\section{CONCLUSION}

The visit to the veterinarian represented in the case of our study by spending just 30 minutes in the waiting room and treatment room without interfering with the dog in any way, is a stress factor requiring adaptive response by activating the body's neuroendocrine system.

Along with endocrine response behavioral manifestations indicated a pattern of stress behavior (hesitation, disregard orders). This is the first indicator that allows noticeable appreciation of discomfort in certain circumstances.

There is a direct correlation between behavioral manifestations in stressful situations and urinary cortisol values.

Determination of urinary cortisol is an important marker in the diagnosis of the state of stress in dogs.

\section{Recommendations}

Status of comfort or discomfort of the animal in the veterinary clinic has a triple significance for the dog, dog owner and veterinarian. For the dog - by maintaining welfare, for medical / veterinary personnel - an animal in a state of agitation, stress

Tab. 2. Urinary cortisol values and stress assessment scale of the studied group

\begin{tabular}{ccccccc}
\hline The subjects & Age & $\begin{array}{c}\text { Reference } \\
\text { values }\end{array}$ & $\begin{array}{c}\text { Urinary cortisol } \\
\text { values obtained } \\
\text { in park }\end{array}$ & $\begin{array}{c}\text { Urinary cortisol } \\
\text { values after visit to } \\
\text { the veterinary clinic }\end{array}$ & $\begin{array}{c}\text { Obtained value on the } \\
\text { stress assessment scale }\end{array}$ \\
\hline 1. & Ruby & 3 years & $1.8-10.9 \mu \mathrm{g} / \mathrm{L}$ & $10,3 \mu \mathrm{g} / \mathrm{L}$ & $27.45 \mu \mathrm{g} / \mathrm{L}$ & 4 points \\
\hline 2. & Szuki & 10 years & $1.8-10.9 \mu \mathrm{g} / \mathrm{L}$ & $4,6 \mu \mathrm{g} / \mathrm{L}$ & $5.01 \mu \mathrm{g} / \mathrm{L}$ & 1 points \\
\hline 3. & Leea & 7 years & $1.8-10.9 \mu \mathrm{g} / \mathrm{L}$ & $9.45 \mu \mathrm{g} / \mathrm{L}$ & $6,6 \mu \mathrm{g} / \mathrm{L}$ & 3 points \\
\hline 4. & Lucky & 7 years & $1.8-10.9 \mu \mathrm{g} / \mathrm{L}$ & $13,5 \mu \mathrm{g} / \mathrm{L}$ & $18,9 \mu \mathrm{g} / \mathrm{L}$ & 3 points \\
\hline 5. & Mini & 5 years & $1.8-10.9 \mu \mathrm{g} / \mathrm{L}$ & $5,8 \mu \mathrm{g} / \mathrm{L}$ & $22,6 \mu \mathrm{g} / \mathrm{L}$ & 5 points \\
\hline 6. & Medi & 7 years & $1.8-10.9 \mu \mathrm{g} / \mathrm{L}$ & $9.45 \mu \mathrm{g} / \mathrm{L}$ & $16,9 \mu \mathrm{g} / \mathrm{L}$ & 4 points \\
\hline
\end{tabular}


can become a danger to those who handle it versus a calm dog and not least for the owner whose opinion is formed according to the welfare of the animal.

Since veterinary medical consultation or other manipulations are indispensable to an animal, we are left merely to effectively anticipate and adapt to stressors. For dogs this can be done by advising the owner regarding the importance of accommodating very young dog with gestures that mimic the medical maneuvers (exam of the mouth, ears, limbs, palpation of different regions).

\section{REFERENCES}

1. Doring D, Roscher A, Scheipl F, Kuchenhoff H, Erhard HM (2009). Fear-related behavior of dogs in veterinary practice. The veterinary journal 182:38-43.

2. Jensen P (2007). Behavioural Biology of Dogs, CAB International.
3. Kelsey AH (2012). The use of cortisol for objective assessment of stress in animals: Pros and cons. Veterinary Journal 192:137-139.

4. Kolevska J, Brunclik V, Svoboda M (2003). Circadian Rhythm of Cortisol Secretion in Dogs of Different Daily Activities. Acta Vet. Brno 72: 599-605.

5. Marcus I (2007). A. Dog Behaviour, Evolution and Cognition, Oxford Univ. Press, New York, 95-100.

6. Mills DS, Braem M, Zulch H (2013. Stress and pheromonatherapy in small animal clinical be- havior, by John Wiley\&sons, Ltd, 242-255.

7. Moberg GP, Mench JA (2000). The biology of animal stress. Basic Principles and Implications for Animal Welfare, CAB International.

8. Overall L K (2013), Manual of Clinical Behavioral Medicine for Dogs and Cats, St. Loui. Missouri: Mosby, Inc.

9. Rusu SA, (2012), Interacțiunea om - animal elemente de etologie aplicată şi terapie asistată de animale, Editura Bybliotek, 151- 172.

10. Stanford TL (1981). Behavior of dogs entering a veterinary clinic. Appl Anim Ethol 7:271-279. 